\title{
Implant Safety Tool Application to Assist the Assessment of Radio-Frequency Radiation Exposure
}

\author{
Piotr Gas' ${ }^{1}$ Arkadiusz Miaskowski ${ }^{*}$ \\ 1 AGH University of Science and Technology, Department of Electrical and Power Engineering, \\ Mickiewicza 30 Avenue, 30-059 Krakow, Poland \\ 2 University of Life Sciences in Lublin, Department of Applied Mathematics and Computer Sciences, \\ Akademicka 13 Street, 20-950 Lublin, Poland \\ * Corresponding author's e-mail: arek.miaskowski@up.lublin.pl
}

\begin{abstract}
This paper discusses a new Implant Safety Tool, used to assess the safety of patients with metal implants exposed to radio-frequency (RF) fields. This specialist tool is discussed on the example of pectus patients with titanium bar-implant inserted during the minimally invasive Nuss procedure. The authors created a $3 \mathrm{D}$ realistic model of a 34-year-old male patient with a Nuss bar-implant. A numerical analysis based on the finite-difference time-domain (FDTD) method was performed for a far field source in the form of a plane wave with the frequency of $64 \mathrm{MHz}$, which corresponds to the RF exposure generated by MRI devices at $1.5 \mathrm{~T}$. The obtained results allow concluding that the concave Nuss bar-implant poses no risk during environmental and occupational RF field exposures.
\end{abstract}

Keywords: Implant Safety Tool, pectus excavatum, metal implant, Nuss procedure, MRI devices, plane wave, FDTD method, RF exposure standards

\section{INTRODUCTION}

The growing number of mobile communication devices [Mazurek et al., 2018], aparatures for medical diagnostics based on electromagnetic (EM) radiation [Gniadek-Olejniczak et al., 2018], as well as sensors for constant real-time monitoring of patients' health [Tayyab et al., 2019], raises widespread concerns about the harmful effects of electromagnetic fields (EMF) for human health [Wyszkowska et al., 2019]. These fears are particularly related to the patients with medical implants, whose number systematically increases both in general public and working environment radio-frequency (RF) field exposures [Zradzinski et al., 2019]. The presence of metal implants in the human body locally strengthens and interferes with the distribution of external EMF [Mat et al., 2017; Yao et al., 2019]. According to many research studies, a high frequency EMFs may affect the tissues of implanted patients, and under certain circumstances may cause harmful implant heating or even thermal damage to adjacent tissues [Destruel et al., 2019; Yao et al., 2019]. This is mainly due to the induction of eddy currents in metal implantable devices under RF fields [Sawicki et al., 2006; Ye et al., 2015]. Both the passive implants, such as vascular stents [Syrek et al., 2017], metal bar-implants [Miaskowski et al., 2016], hip and orthopedic implants [Kozlov et al., 2015; Miaskowski et al., 2012], prosthetic and dental implants [Safari et al., 2016], as well as the active implants like pacemakers and cardiac implantable devices [Gniadek-Olejniczak et al., 2018; Smondrk et al., 2018], deep brain stimulators [Guerin et al., 2019], etc., interact with the external EMFs produced by communication systems [Mat et al., 2017; Ye et al., 2015] or strong magnetic gradients generated by modern medical diagnostic tools such as magnetic resonance imaging (MRI) devices [Gniadek-Olejniczak et al., 2018; Yao et al., 2019]. Metal implants often prevent their patients from enjoying the benefits of modern civilization including MRI due to severe 
interactions with active implantable medical devices (AIMD) or discomfort feeling, pain and burning sensations [Zradzinski et al., 2019]. The harmful health effects related to the radiation of MRI devices depend on its frequency, polarization and distribution in space and time. However, compliance with stringent safety protocols of MR imaging guarantee the highest precautions during diagnostic procedures and confirmed the feasibility of MRI examinations even in the case of cardiac implantable electronic devices [Gniadek-Olejniczak et al., 2018]. What is more, in MRI scans, characteristic bright spots appear at the implant location, which can disturb the correct diagnosis of the health condition of the adjacent tissues [Destruel et al., 2019]. It is worth noting that the patients' safety and health risk during MRI scanning at $128 \mathrm{MHz}(3.0 \mathrm{~T})$ has not yet been established and the existing reports mainly relate to the MRI devices operating at $64 \mathrm{MHz}$ (1.5 T) [Guerin et al., 2019].

One of the methods for assessing the exposure to high-frequency EM radiation is estimating the specific absorption rate (SAR), which determines the amount of the EM energy absorbed by the unit mass of an object [Destruel et al., 2019; Gas et al., 2019; Jovanovic et al., 2019; Mat et al., 2017; Miaskowski et al., 2016; Smondrk et al., 2018]. A risk evaluation of medical implants under RF fields may be also connected with temperature increase of tissue in the vicinity of a metal implant [Yao et al., 2019]. A computer modeling of the EMFs, RF-induced heating of medical implants and surrounding tissues is an important element of implanted patients' safety investigations. This paper discusses a new Implant Safety Tool, used to assess the safety of patients with metal implants exposed to RF fields. This specialist tool is discussed on the example of the pectus patients with titanium bar-implants inserted during the minimally invasive Nuss procedure.

\section{Chest Wall Deformations and Their Treatment}

The chest wall deformities such as pectus excavatum (PE) [Ewais et al., 2019] and pectus carinatum (PC) [Muntean et al., 2018] are popular disorders of the skeletal system (see Figure 1). Thoracic costal cartilage may undergo abnormal inherited deformations, which occur especially in children and adolescents. The cause of such disorders is still not fully understood. Specific weakness of the sternocostal cartilage and costal matrix disorganization may be explained by the lack of cartilage vascularization, persistent hypoxia and the acidic external environment of cartilage chondrocytes [Asmar et al., 2019]. Approximately $90 \%$ of the thoracic abnormalities (one case in every 434 live births) is related to pectus excavatum also called funnel chest or sunken chest [Ewais et al., 2019]. This malformation is characterized by deep depression of the sternum and the neighboring ribs into the interior of thoracic cavity, which also affects the abnormal deformity of the costal arch. Such distortion manifests itself at any stage of life, especially during childhood and puberty, when an excessive growth of bony thoracic skeleton emphasizes a defect.

The opposite dysfunction to PE is so-called pectus carinatum also known as keel chest or pigeon chest [Muntean et al., 2018]. It involves an abnormal protrusion of the sternum with the

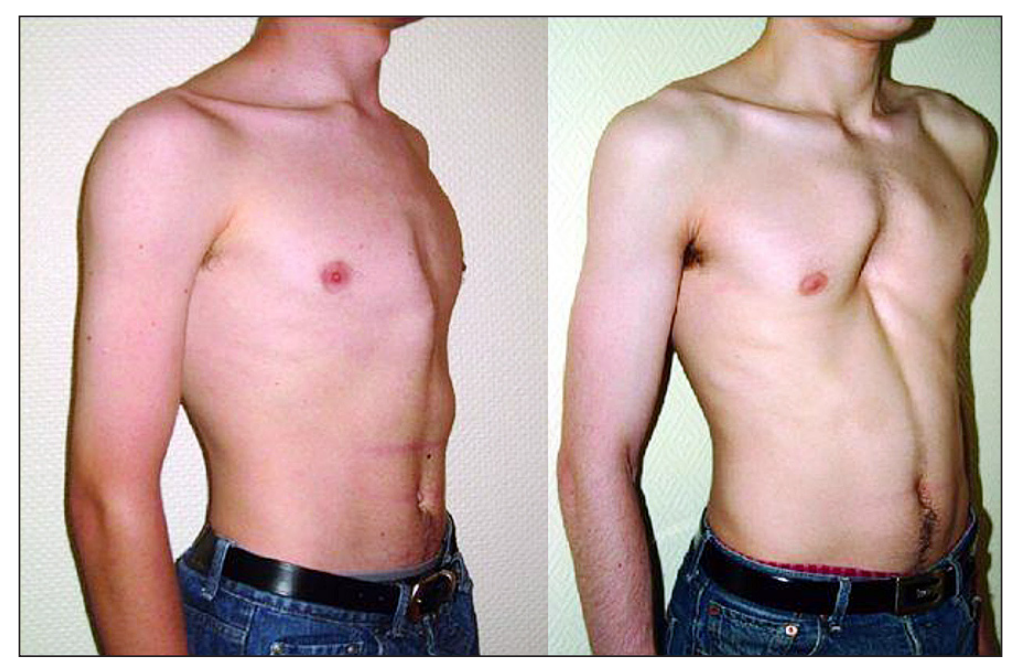

Figure 1. Adolescent boys with pectus carinatum (left) and pectus excavatum (right) [Thoracic Surgery, 2019] 
adjacent ribs towards the outside of the body. It occurs about nine times less than funnel chest and affects no more than $5-7 \%$ of all thorax anomalies. What is interesting, most cases of PC deformations are identified in male teenagers as their skeleton grows. Pigeon chest is less common than the sunken chest and mainly affects males [Muntean et al., 2018]. It should be stressed that the pectus malformations of the skeletal system reduce the capacity of the lungs and consequently lead to the disorders of the cardiopulmonary system that can manifest with such symptoms as recurrent coughing, fatigability, dyspnea, dizziness, fainting and even bronchitis or pneumonia. In addition, the pectus-related severe cardiopulmonary implications increase with the patient's age [Ewais et al., 2019].

Serious congenital deformities of the chest wall can be treated using a variety of surgical and non-surgical techniques. Surgical correction is required in the symptomatic cases of severe circulatory-respiratory failure caused by strong sternum deformity [Ewais et al., 2019]. In recent times, the so-called minimally invasive Nuss method, using appropriately profiled bar-implants under the sternum is widely used [Jaroszewski et al., 2018] (see
Figure 2). By providing adequate pressure on the costal cartilage of the sternum, their task is to restore the natural shape of thoracic cage. Nuss bars have only corrective functions and are implanted in the patient's trunk for a period of 2-3 years (see Figure 3). They usually interact mechanically on the thoracic costal cartilages with a maximum force up to $250 \mathrm{~N}$ [Betti et al., 2014]. What is important, a Nuss bar-implant displacement may occur during the implantation period; thus, the continuous real-time monitoring of the progressive thoracic wall correction is necessary, which can detect health problems in advance, provide better treatment for the pectus patients, and determine the best time to remove the metal implant [Wang et al., 2017]. Moreover, the titanium or other alloys are mainly utilized for the production of such concave implants adapted to the patient's anthropometric features [Miaskowski et al., 2016].

Until recently, in the surgeon community there was a belief that the use of the Nuss method in adults raises a lot of controversy, but now there are many reports of effective chest wall repairing even for the 50-year-old adult patients [Ewais et al., 2019]. However, in the case of adults, the original Nuss procedure requires

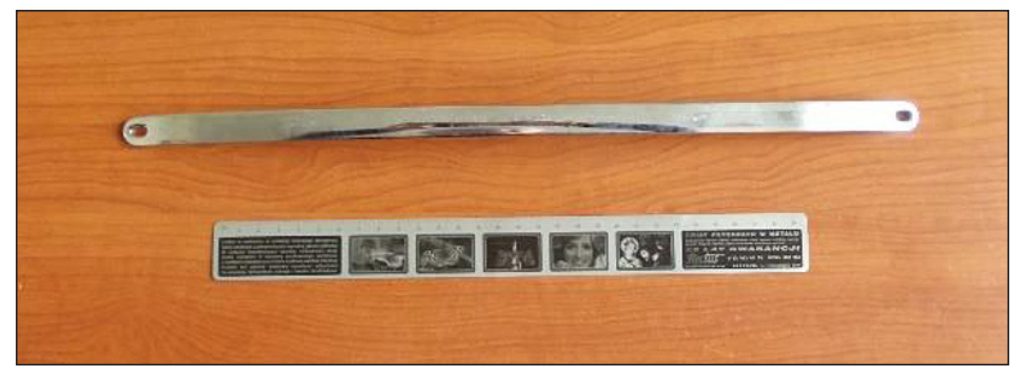

Figure 2. The practically used titanium Nuss bar and the ruler [Miaskowski et al., 2016]

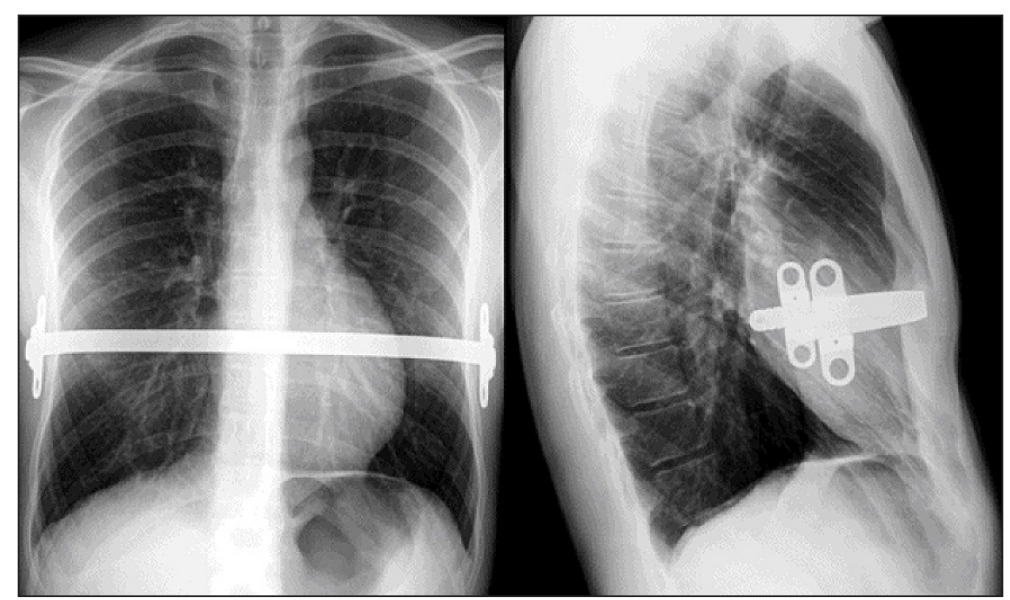

Figure 3. A Nuss bar implanted in the patient's wall chest [Thoracic Surgery, 2019] 
some modifications [Jaroszewski et al., 2018]. A 15 year-experience in Nuss surgical correction of 239 patients aged between 14-34 are summarized in the study [Cierpikowski et al., 2018]. The authors observed beneficial long-term effects in $96.7 \%$ of the pectus patients, and postoperative complications in $16.9 \%$ of them, mostly in the adult patients with asymmetric chest deformations. Importantly, no cases of perioperative mortality were found. The technical refinements in the Nuss procedure have significantly reduced severe complications in the adult PE patients [Ewais et al., 2019]. Moreover, the recurrence rate after bar-implant removal ranged from 0 to $33 \%$, and it increases inversely with the period of bar-implant [Farronato et al., 2019]. Most of the patients' satisfaction questionnaires after the Nuss method showed an improvement of selfesteem in the PE patients. Similar treatment procedures, namely minimal access repair for pectus carinatum, were developed for the PC patients, whose titanium bar-implant is localized over the sternum [Muntean et al., 2018].

The choice of the appropriate method of treating chest dysfunctions depends on the type and level of trunk bone deformity and the individual physiological abilities of the pectus patient. In order to determine the degree of chest deformation, support from medical imaging and achievements of reverse engineering are often needed to create specialized Nuss implant computer-aided systems [Kim et al., 2019]. In [Antonowicz et al., 2016], based on the 3D CT scans of the skeletal system, the authors demonstrated the model and developed a prototype of an external stabilizer supporting the treatment of pectus carinatum in young men. Other researchers [Betti et al., 2014] proposed advanced sensorized Nuss bar prototype to monitor changes in pressure (forces) acting on the chest wall of the PE patients during the period of bar-implant. Owing to such device, the treatment time is limited to the minimum necessary to achieve lasting pectus correction, and thus the probability of negative tissue-implant interactions is reduced. Another similar tool, namely flexible microfluidic-based sensor for Nuss bar motion monitoring, was developed by [Wang et al., 2017]. In another study [Huang et al., 2019], the authors gave the advantages of the 3D printed model-assisted Nuss procedure. On the basis of CT images and 3D printing, they created a model of an appropriately bent metal implant, perfectly matching the patient's anatomy. The results of the treatment designed in this way for 15 pectus patients were compared with 342-patient group treated with traditional Nuss procedure. The proposed solution reduces the time of surgery and costs associated with the correction of curvature pectus bar-implant in relation to standard treatment. A similar solution for patient-specific Nuss bars aided with computer design (CAD) and manufacturing (CAM) was proposed in [Kim et al., 2019]. Although the procedure for producing bar-implants is complicated, it can reduce the failure rate of the Nuss treatment and increase the level of the patient's satisfaction. Additionally, in [Rechowicz et al., 2015], the authors describe the Nuss procedure surgical simulator as a valuable tool of training among medical students and young surgeons. These types of virtual exercises are intended to limit the basic errors made by medical practitioners during the actual surgery of Nuss correction.

\section{METHODS}

The interaction issue of a concave Nuss barimplant, placed in the body of a young adult patient, with the RF field produced by the MRI device was solved numerically based on the Finite Difference Time Domain (FDTD) introduced by K.S. Yee in the middle of the previous century [Yee, 1966]. It provides a direct solution to the Maxwell's equations in the time domain, namely [Gas et al., 2019]:

$$
\begin{gathered}
\nabla \times \mathbf{H}=\frac{\partial}{\partial t} \varepsilon \mathbf{E}+\sigma \mathbf{E} \\
\nabla \times \mathbf{E}=\frac{\partial}{\partial t} \mu \mathbf{H}-\sigma_{\mathrm{H}} \mathbf{H}
\end{gathered}
$$

where: $\varepsilon=\varepsilon_{0} \varepsilon_{\mathrm{r}}$ and $\mu=\mu_{0} \mu_{\mathrm{r}}$ are the electric permittivity and magnetic permeability of given medium, respectively.

$\varepsilon_{0}=8.85 \cdot 10^{-12} \mathrm{~F} / \mathrm{m}$ and $\mu_{0}=4 \pi \cdot 10^{-7} \mathrm{H} / \mathrm{m}$ mean electric and magnetic constants, and $\varepsilon_{\mathrm{r}}$ and $\mu_{\mathrm{r}}$ correspond to dimensionless relative quantities for $\varepsilon$ - and $\mu$-parameters. $\sigma(\mathrm{S} / \mathrm{m})$ and $\sigma_{\mathrm{H}}(\Omega / \mathrm{m})$ stand for the electric conductivity and magnetic conductivity (permeance) of a conducting medium, associated with electrical and magnetic losses. 
In the FDTD method, the vector components of electric $\mathbf{E}(\mathrm{V} / \mathrm{m})$ and magnetic $\mathbf{H}(\mathrm{A} / \mathrm{m})$ field strengths are arranged in a rectangular coordinate system $(x, y, z)$ on shifted grids as shown in Figure 4. A single cubic element of such mesh is called a Yee cell [Yee, 1966]. The $E$-field components are located in the middle of the edge and the $H$-field components are placed in the centre of the faces of the Yee cell. The equations above are discretized in space and time by means of second order approximation with finite differences, as follows [Gas et al., 2019]:

$$
\begin{gathered}
\frac{\partial E(i, j, k, n)}{\partial x}= \\
=\frac{E^{n}(i+1 / 2, j, k, n)-E^{n}(i-1 / 2, j, k, n)}{\Delta x}+ \\
+O\left[(\Delta x)^{2}\right] \\
\frac{\partial E(i, j, k, n)}{\partial t}= \\
=\frac{E^{n+1 / 2}(i, j, k)-E^{n-1 / 2}(i, j, k)}{\Delta t}+O\left[(\Delta t)^{2}\right]
\end{gathered}
$$

where: $E^{n}$ means the electric field components at discrete time values $t=n \Delta t$, and $i, j, k$ are the spatial cubic grid indices (see Figure 4), $n$ stands for a temporal index and $\Delta t$ is a discrete time step. $O\left[(\Delta x)^{2}\right]$, $O\left[(\Delta t)^{2}\right]$ are second order errors introduced by truncating the Taylor series for the determined derivatives of the first row, and $\Delta x$ denotes a grid discretization step along the $x$-axis.

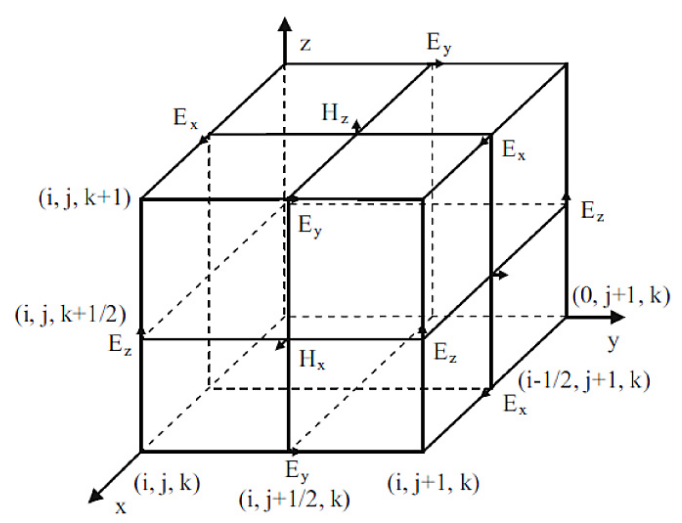

Figure 4. A cubic Yee cell with marked EM field components
Similar derivatives to equation (3) can be obtained for other Cartesian coordinates. According to presented notation of finite differences, the Maxwell's equations for the $E_{x}$-component can be written as [Osaci, 2018]:

$$
\begin{gathered}
\frac{\left.E_{x}\right|_{i, j, k} ^{n+1}-\left.E_{x}\right|_{i, j, k} ^{n}}{\Delta t}=. \\
=\frac{1}{\varepsilon_{i, j, k}}\left(\frac{\left.H_{z}\right|_{i, j+1 / 2, k} ^{n+1 / 2}-\left.H_{z}\right|_{i, j-1 / 2, k} ^{n+1 / 2}}{\Delta y}-\right. \\
\left.-\frac{\left.H_{y}\right|_{i, j, k+1 / 2} ^{n+1 / 2}-\left.H_{z}\right|_{i, j, k-1 / 2} ^{n+1 / 2}}{\Delta z}-\left.\sigma_{i, j, k} E_{x}\right|_{i, j, k} ^{n+1 / 2}\right)
\end{gathered}
$$

Next, using the semi-implicit approximation in arithmetic meaning:

$$
\left.E_{x}\right|_{i, j, k} ^{n+1 / 2}=\frac{\left.E_{x}\right|_{i, j, k} ^{n+1}+\left.E_{x}\right|_{i, j, k} ^{n}}{2}
$$

the following equation can be given [Osaci, 2018]:

$$
\begin{gathered}
\left.E_{x}\right|_{i, j, k} ^{n+1}=\left.\left(\frac{1-\frac{\Delta t \sigma_{i, j, k}}{2 \varepsilon_{i, j, k}}}{1+\frac{\Delta t \sigma_{i, j, k}}{2 \varepsilon_{i, j, k}}}\right) E_{x}\right|_{i, j, k} ^{n}+\left(\frac{1-\frac{\Delta t \sigma_{i, j, k}}{2 \varepsilon_{i, j, k}}}{1+\frac{\Delta t \sigma_{i, j, k}}{2 \varepsilon_{i, j, k}}}\right) \\
\left(\frac{\left.H_{z}\right|_{i, j+1 / 2, k} ^{n+1 / 2}-\left.H_{z}\right|_{i, j-1 / 2, k} ^{n+1 / 2}}{\Delta y}-\frac{\left.H_{y}\right|_{i, j, k+1 / 2} ^{n+1 / 2}-\left.H_{z}\right|_{i, j, k-1 / 2} ^{n+1 / 2}}{\Delta z}\right)
\end{gathered}
$$

Analogous expressions can be determined for all six field components. Importantly, to obtain a stable solution, the time step must be limited according to the Courant-Friedrich-Levy (CFL) criterion [Yee, 1966]:

$$
\begin{gathered}
\Delta t \leq \frac{1}{c_{\max } \sqrt{\frac{1}{(\Delta x)^{2}}+\frac{1}{(\Delta y)^{2}}+\frac{1}{(\Delta z)^{2}}}}, \\
c_{\max }=\frac{1}{\sqrt{\mu \varepsilon}}
\end{gathered}
$$

where: $c_{\max }$ is the maximum speed of light in a given medium.

From equation (7) it can be seen that $\Delta t$-parameter depends on the Yee cell size and impacts on the computational requirements of the simulation. 
In the dosimetric calculations involving tissue-implant iteractions under RF fields, special attention is paid to including the anthropomorphic 3D virtual models of human body in computer simulation. Using the possibilities of FDTDbased commercial software Sim4Life [Sim4Life, 2019], the authors created a realistic 3D model of a 34-year-old male patient with a Nuss bar-implant. For this purpose, a Duke human phantom [Duke, 2019] belonging to the Virtual Population family was modified. It is a voxel model that well reflects the complex anatomical features and tissue heterogeneity with resolution of $0.5 \mathrm{~mm}$. Such high resolution takes place at a significant computational cost. The number of voxels is determined by the geometric features of the model. Originally, the Duke model, with height of $177 \mathrm{~cm}$, weight of $70.2 \mathrm{~kg}$ and the body mass index (BMI) value equal to $22.4 \mathrm{~kg} \cdot \mathrm{m}^{-2}$, includes up to 305 different tissues, organs and structures. For the computational reasons, the numerical model was limited to the trunk of young adult (Figure 5) including 20 main tissue structures listed in Table 1. The dielectric characteristics of tissues taken from the IT'IS Database [Hasgall et al., 2018] are based on the Gabriel's dispersive model of human tissues for the excitation frequency of $64 \mathrm{MHz}$. The Nuss-bar model implanted in chest of young adult is shown in Figure 6. A numerical analysis based on FDTD method was performed for a far field source in the form of a plane wave with the frequency of $64 \mathrm{MHz}$, which corresponds to the RF exposure generated by the MRI devices at $1.5 \mathrm{~T}$. Electric field $\mathbf{E}$ of unit magnitude, $1 \mathrm{~V} / \mathrm{m}$, was directed perpendicular to the longitudinal axis of the body and parallel to the implant axis, magnetic field $\mathbf{H}$ downwards, and the propagation constant $\mathbf{k}$ towards the modeled body. In the current investigation, the metal bar-implant was considered as a perfect electric conductor (PEC). Furthermore, the open boundary conditions were assumed to simulate six perfectly matched layers (PML) at the external boundaries of computational domain.

\section{Implant Safety Tool and Obtained Results}

The Implant Safety Tool (IMSAFE) was created by the scientific community [Sim4Life, 2019] to assess the safety of patients with medical implants during the MRI exposition, in accordance with the ISO/TS 10974 standard [ISO/TS 10974:2018; Yao et al., 2019]. This tool takes into account the technical aspects of the MRI devices (i.e. field

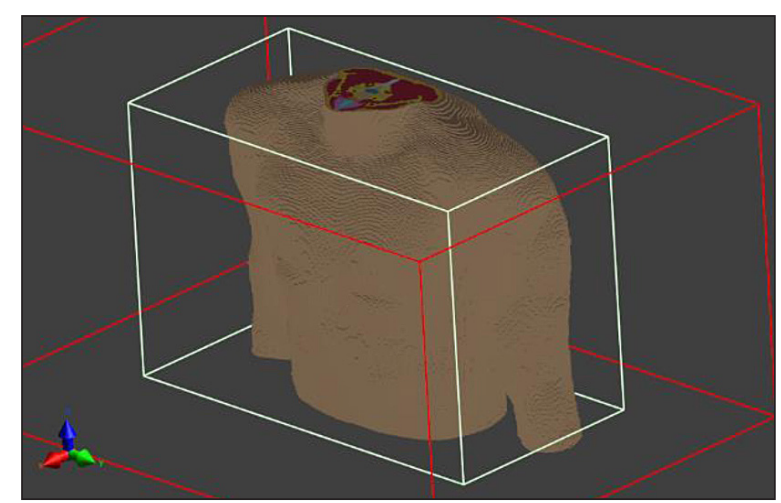

Figure 5. A trunk phantom used in the simulation (red indicates the computational domain)

excitation frequency, coil design), as well as the individual characteristics of the patient's body (BMI, age, gender), its location inside the MRI coils, as well as the type of implant used. What is more, the IMSAFE application allows creating any volume of an enlarged implant using splines and perpendicular cylindrical discs of various radii and lengths with specified length deviation and resolution. The user defines the implant baseline and the program generates a series of random lines within individual disks located in the target region of interest (ROI). Therefore, the IMSAFE software allows simulating the metal implant using so-called implant routes given in selected volume (see Figures 7) and there is no need to define the dielectric parameters of the titanium implant, which is treated by default as a perfect electric conductor (PEC). This procedure significantly reduces the usage of computational memory and time of numerical calculations. What is important, the performed in silico simulation assumed 50 IMSAFE lines (see Figure 8) with average length of $31.41 \mathrm{~cm}$, which determines the

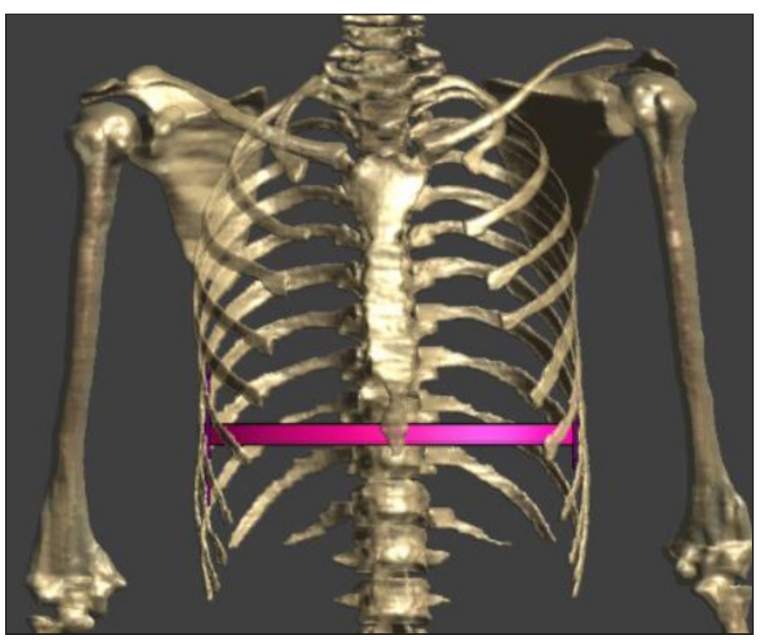

Figure 6. Modeled Nuss bar inside the human chest 
Table 1. Dielectric propertis of tissues included in the modelled male trunk for frequency of $64 \mathrm{MHz}$

\begin{tabular}{|l|c|c|l|c|c|}
\hline \multicolumn{1}{|c|}{ Tissues/Organs } & $\begin{array}{c}\text { Permittivity } \\
\varepsilon_{r}(-)\end{array}$ & $\begin{array}{c}\text { Conductivity } \\
\sigma(\mathrm{S} / \mathrm{m})\end{array}$ & \multicolumn{1}{c|}{ Tissues/Organs } & $\begin{array}{c}\text { Permittivity } \\
\varepsilon_{r}(-)\end{array}$ & $\begin{array}{c}\text { Conductivity } \\
\sigma(\mathrm{S} / \mathrm{m})\end{array}$ \\
\hline blood & 86.44 & 1.207 & liver & 80.56 & 0.448 \\
\hline bone & 30.87 & 0.161 & lung & 37.10 & 0.289 \\
\hline bronchi & 58.89 & 0.528 & muscle & 72.23 & 0.688 \\
\hline cartilage & 62.91 & 0.452 & skin & 92.17 & 0.436 \\
\hline fat & 13.64 & 0.066 & small intestine & 118.36 & 1.591 \\
\hline gallbladder & 87.40 & 0.966 & spinal cord & 55.06 & 0.312 \\
\hline heart muscle & 106.51 & 0.678 & spleen & 110.56 & 0.744 \\
\hline intervertebral disc & 51.46 & 0.843 & stomach & 85.82 & 0.878 \\
\hline kidney & 118.56 & 0.741 & thymus & 55.81 & 0.637 \\
\hline large intestine & 94.66 & 0.638 & vertebrae & 16.68 & 0.060 \\
\hline
\end{tabular}

length of the concave Nuss-bar implant. Using the IMSAFE application, the tangential components of the electric field strength along the implant routes, as well as electric and magnetic field distributions at any intersections of the computational area can be easily obtained as shown in the Figures 7-9.

As shown in Figures 7 and 8, the largest values of the tangential component of the electric field strength $\mathbf{E}_{\tan }$ along the implant routes occur in the middle of the bent implant near the sternum, where they do not exceed a value of $0.318 \mathrm{~V} / \mathrm{m}$, as well as on the implant stabilizers $(0.334 \mathrm{~V} / \mathrm{m}$ - left and $0.307 \mathrm{~V} / \mathrm{m}$ - right $)$ that are perpendicular to the incident $\mathbf{E}$ wave. What is more, the highest values of $E_{\mathrm{RMS}}$ on the plane normal to the Nuss bar-implant are observed in the area of the limbs and between limbs and trunk as shown in Figure 9. The electric field distribution on the same slice for $E_{\mathrm{RMS}}$-values averaged to $10 \mathrm{~g}$ of human tissue $\left(E_{\mathrm{RMS}, 10 \mathrm{~g}}\right)$ for $6 \mathrm{~min}$ period exposure in accordance to the standard [ISO/TS 10974:2018] is depicted in Figure 10. In this case, the highest field values of $0.247 \mathrm{~V} / \mathrm{m}$ were observed in the vinicity of the left limb. The exact $E_{\mathrm{RMS}}$-values in the case of a model with a metal implant and without it are gathered together in Table 2. As demonstated, the presence of the implant in the far RF field of $64 \mathrm{MHz}$ causes a slight increase in the E-field intensity. Table 3 compares the reference levels [1999/519/EC] and the action levels [2013/35/EU], respectively for environmental and occupational exposures in the presence of electric component of RF radiation $(100 \mathrm{kHz}-300 \mathrm{MHz})$. As the frequency increases, the $E_{\mathrm{RMS}}$ values decrease, and for the excitation frequency $64 \mathrm{MHz}$, which corresponds to the MRI exposure, they are $28 \mathrm{~V} / \mathrm{m}$ for general public and $61 \mathrm{~V} / \mathrm{m}$ for workers exposition, respectively.

It should be noted that the E-field exposure limits provided both by recommendation [1999/519/EC] and less restrictive Directive [2013/35/EU] are many times higher than the ones obtained for the analyzed phantoms with the

Table 2. Maximum effective values of electric field for the models with the titanium bar and without it

\begin{tabular}{|c|c|}
\hline \multicolumn{2}{|c|}{ Maximum values of $E_{\mathrm{RMS}}(\mathrm{V} / \mathrm{m})$} \\
\hline model without implant & model with implant \\
\hline 3.51 & 3.53 \\
\hline
\end{tabular}

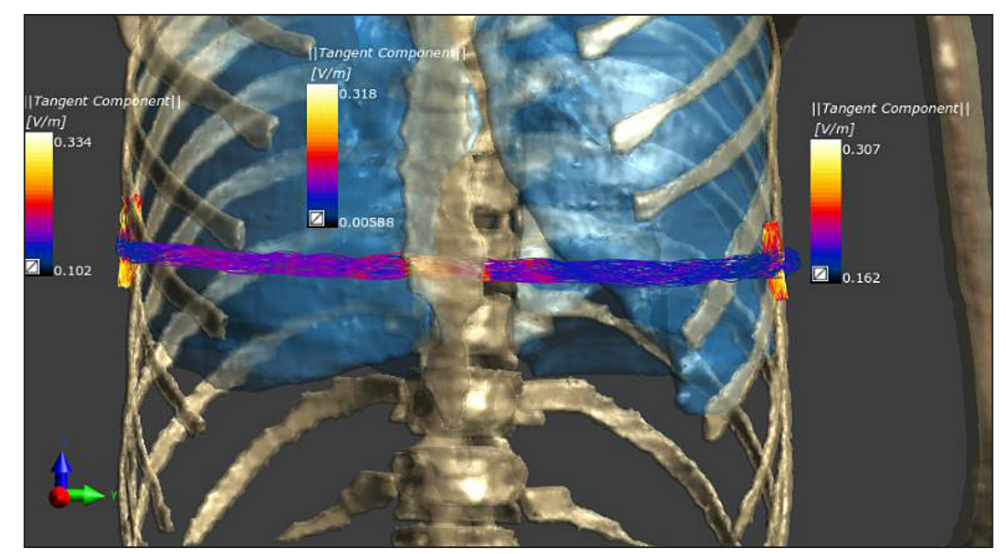

Figure 7. Tangent component of electric field strength on splines generated inside the Nuss bar 


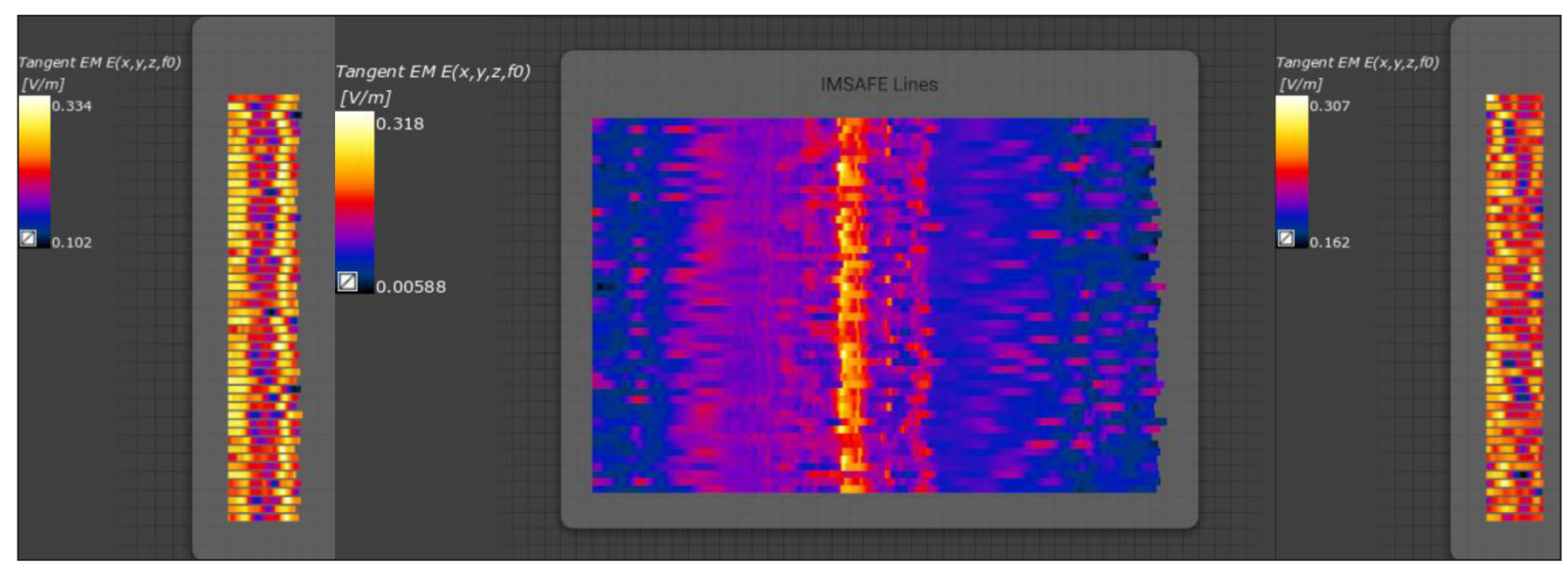

Figure 8. Tangential $E$-field distributions along IMSAFE lines generated on implant stabilizers (left and right) as well as in the main part of Nuss bar-implant (center)

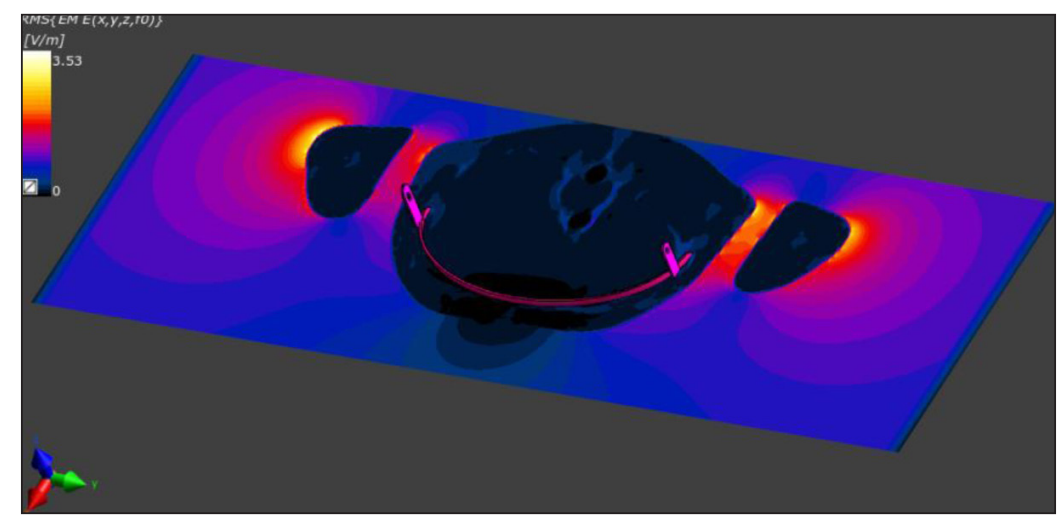

Figure 9. Effective value of electric field strength $\left(E_{\mathrm{RMS}}\right)$ on the slice perpendicular to the bar-implant

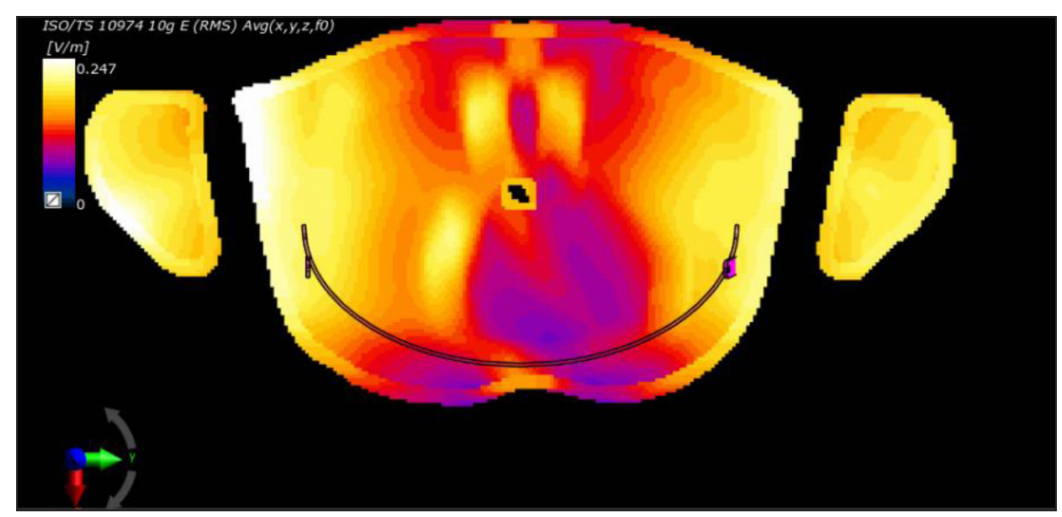

Figure 10. Averaged electric field values $\left(E_{\mathrm{RMS}, 10 \mathrm{~g}}\right)$ on the slice normal to the titanium bar

Table 3. Limit levels of exposures for general public and workers in RF fields ( $100 \mathrm{kHz}-300 \mathrm{MHz})$

\begin{tabular}{|c|c|c|c|}
\hline \multicolumn{2}{|c|}{ Environmental Exposure [1999/519/EC] } & \multicolumn{2}{c|}{ Occupational Exposure [2013/35/EU] } \\
\hline Frequency range & $E_{\mathrm{RMS}}(\mathrm{V} / \mathrm{m})$ & Frequency range & $E_{\mathrm{RMS}}(\mathrm{V} / \mathrm{m})$ \\
\hline $100 \mathrm{kHz} \leq f<1 \mathrm{MHz}$ & 87 & $100 \mathrm{kHz} \leq f<1 \mathrm{MHz}$ & $6.1 \times 10^{2}$ \\
\hline $1 \mathrm{MHz} \leq f<10 \mathrm{MHz}$ & $87 / f^{1 / 2}$ & $1 \mathrm{MHz} \leq f<10 \mathrm{MHz}$ & 61 \\
\hline $10 \mathrm{MHz} \leq f<300 \mathrm{MHz}$ & 28 & $10 \mathrm{MHz} \leq f<300 \mathrm{MHz}$ & $f$ is the frequency expressed in Hz \\
\hline \multicolumn{2}{|r|}{$f$ as indicated in the frequency range column } & \multicolumn{2}{|c}{} \\
\hline
\end{tabular}


titanium built-implant and without Nuss bar (see Tables 2 and 3). Thus, the concave Nuss bar-implant poses no risk during the environmental and occupational RF field exposures.

\section{CONCLUSIONS}

The need for the access to modern medical imaging tools (MRI) also applies to the pectus patients with titanium Nuss bar implanted in the chest. The interaction of the metal implant with the external RF field can pose danger to the patients' health, due to the induction of eddy currents in the Nuss bar-implant and its possible harmful heating, discomfort, pain and burning. That is why researchers create $3 \mathrm{D}$ realistic human models to assess the safety of RF radiation exposure similar to the one presented in this paper. To summarize the presented in silico results for numerical phantom of 34-yearold male with Nuss bar-implant, the following conclusions could be drawn. The simplification of implant modeling using IMSAFE lines reduces the required computational memory of computer and the calculation time for anatomically correct 3D human models. The obtained results clearly show that there is no significant threat for the pectus patients with implanted Nuss bars exposed to the far field of MRI devices at $64 \mathrm{MHz} / 1.5 \mathrm{~T}$. The simulated $E_{\mathrm{RMS}}$-values for models with and without concave implant in excess comply the ICNIRP and EU guidelines both for occupational and environmental RF field exposures. The risk analysis of the titanium Nuss bar-implants in the near field of MRI coils requires further analysis. It should be emphasized that the threat assessment presented by the authors with the Implant Safety Tool application has never been presented in the literature of subject before.

\section{REFERENCES}

1. Antonowicz M., Kajzer A., Kajzer W. 2016. Application of reverse engineering in supporting the treatment of pectus carinatum. Conference of Information Technologies in Biomedicine, 217-225.

2. Asmar A., Semenov I., Kelly Jr R., Stacey M. 2019. Abnormal response of costal chondrocytes to acidosis in patients with chest wall deformity. Experimental and Molecular Pathology, 106, 27-33.

3. Betti S., Ciuti G., Ricotti L., Ghionzoli M., Cavallo F., Messineo A., Menciassi A. 2014. A sensorized Nuss bar for patient-specific treatment of Pectus Excavatum. Sensors, 14(10), 18096-18113.
4. Cierpikowski P., Rzechonek A., Blasiak P., Lisowska H., Pniewski G., Le Pivert P. 2018. Surgical Correction of Pectus Excavatum by the Nuss Procedure: A 15-Year Experience Study. Clinical Investigation, 31-40.

5. Destruel A., Fuentes M., Weber E., O’Brien K., Jin J., Liu F., Markus B., Crozier S. 2019. A numerical and experimental study of RF shimming in the presence of hip prostheses using adaptive SAR at $3 \mathrm{~T}$. Magnetic Resonance in Medicine, 81(6), 3826-3839.

6. Ewais M.M., Chaparala S., Uhl R., Jaroszewski D.E. 2018. Outcomes in adult pectus excavatum patients undergoing Nuss repair. Patient Related Outcome Measures, 9, 65.

7. Farronato A., Ghionzoli M., Messineo A., Politi L., Divisi D., Gonfiotti A., Crisci R. 2019. Pectus excavatum in adolescents and children: the Nuss technique. Pediatric Medicine, 2, 32.

8. Gas P., Miaskowski A. 2019. SAR optimization for multi-dipole antenna array with regard to local hyperthermia. Przeglad Elektrotechniczny, 95(1), 17-20. DOI: 10.15199/48.2019.01.05

9. Gniadek-Olejniczak K., Makowski K., Olszewski A., Tomczykiewicz K., Krawczyk A., Mroz J. 2018. State-of-the-art approach towards magnetic resonance imaging of the nervous system structures in patients with cardiac implantable electronic devices. Neurologia i Neurochirurgia Polska, 52(6), 652-656.

10. Guerin B., Iacono M.I., Davids M., Dougherty D.D., Angelone L.M., Wald L.L. 2019. The" virtual DBS population": five realistic computational models of deep brain stimulation patients for electromagnetic MR safety studies. Physics in Medicine and Biology, 64(3), 035021.

11. Hasgall P.A., Di Gennaro F., Baumgartner C., Neufeld E., Lloyd B., Gosselin M.C., Payne D., Klingenbock A., Kuster N. 2018. IT'IS Database for thermal and electromagnetic parameters of biological tissues, Version 4.0. DOI: 10.13099/ VIP21000-04-0

12. Huang Y.J., Lin K.H., Chen Y.Y., Wu T.H., Huang H.K., Chang H., Lee S.C., Chen J.E., Huang T.W. 2019. Feasibility and Clinical Effectiveness of Three-Dimensional Printed Model-Assisted Nuss Procedure. The Annals of Thoracic Surgery, 107(4), 1089-1096.

13. ISO/TS 10974:2018 Assessment of the safety of magnetic resonance imaging for patients with an active implantable medical device

14. Jovanovic D.B., Stankovic V., Cvetkovic N.N., Krstic D., Vuckovic D. 2019. The impact of human age on the amount of absorbed energy from mobile phone. COMPEL-The International Journal for Computation and Mathematics in Electrical and Electronic Engineering, 38(5), 1465-1479. 
15. Jaroszewski D.E., Velazco C.S. 2018. Minimally invasive pectus excavatum repair (MIRPE). Operative Techniques in Thoracic and Cardiovascular Surgery, 23(4), 198-215.

16. Kim Y.J., Heo J.Y., Hong K.H., Lim B.Y., Lee C.S. 2019. Computer-aided design and manufacturing technology for identification of optimal nuss procedure and fabrication of patient-specific nuss bar for minimally invasive surgery of pectus excavatum. Applied Sciences, 9(1), 42.

17. Kozlov M., Noetscher G.M., Nazarian A., Makarov S.N. 2015. Comparative analysis of different hip implants within a realistic human model located inside a 1.5 T MRI whole body RF coil. 2015 37th Annual International Conference of the IEEE Engineering in Medicine and Biology Society (EMBC), 7913-7916. DOI: 10.1109/EMBC.2015.7320227

18. Mat M.H., Jusoh M., Rahim H., Yusoff M.I. 2017. A brief review of the emf interaction: metal implantation and biological tissues. Advanced Science Letters, 23(6), 5565-5568.

19. Mazurek P.A., Michałowska J., Koziel J., Gad R., Wdowiak A. 2018. The intensity of electromagnetic fields in the range of GSM 900, GSM 1800 DECT, UMTS, WLAN in built-up areas. Przeglad Elektrotechniczny, 2018, 94(12), 202-205.

20. Miaskowski A., Gas P., Krawczyk A. 2016. SAR Calculations for titanium bar-implant subjected to microwave radiation. 17th International Conference Computational Problems of Electrical Engineering (CPEE), 1-4. DOI:10.1109/CPEE.2016.7738726

21. Miaskowski A., Krawczyk A., Ishihara Y. 2012. A numerical evaluation of eddy currents distribution in the human knee with metallic implant. COMPEL-The International Journal for Computation and Mathematics in Electrical and Electronic Engineering, 31(5), 1441-1447.

22. Muntean A., Stoica I., Saxena A.K. 2018. Pigeon chest: comparative analysis of surgical techniques in minimal access repair of pectus carinatum (MARPC). World Journal of Pediatrics, 14(1), 18-25.

23. Osaci M. 2018. Numerical simulation methods of electromagnetic field in higher education: didactic application with graphical interface for FDTD method. International Journal of Modern Education and Computer Science, 10(8), 1-10

24. Rechowicz K.J., Obeid M.F., Chemlal S., McKenzie F.D. 2015. Simulation of the critical steps of the Nuss procedure. Computer Methods in Biomechanics and Biomedical Engineering: Imaging \& Visualization, 3(4), 189-203.

25. Safari M., Abdolali A. 2016. Dental Implants and Mobile-Phone Use: How implant presence and position affect antenna parameters, specific absorption rate, and current density. IEEE Antennas and Propagation Magazine, 58(5), 43-51.

26. Sawicki B., Starzynski J., Wincenciak S. 2006. Numerical model of magnetic stimulation with metal implants. IEEE Transactions on Magnetics, 42(4), 783-786.

27. Smondrk M., Benova M., Psenakova Z. 2018.
Evaluation of SAR in human body model comprising of implanted pacemaker. 2018 ELEKTRO, 1-5. DOI: 10.1109/ELEKTRO.2018.8398336

28. Syrek P., Skowron M. 2017. The impact of overhead lines for employees with stents, IOP Conference Series-Materials Science and Engineering, 200, 012013.

29. Tayyab M., Sharawi M.S., Shamim A., Al-Sarkhi A. 2019. A low complexity RF based sensor array for lung disease detection using inkjet printing. International Journal of RF and Microwave ComputerAided Engineering, 29(4), e21586.

30. Wang D., Yang Y., Vahala L., McKenzie F.D., Hao Z. 2017. Aflexible microfluidic-based sensor for monitoring the bending and tilting of a metal bar for pectus excavatum (PE) patients. ASME 2017 International Mechanical Engineering Congress and Exposition, 1-6.

31. Wyszkowska J., Jankowska M., Gas P. 2019. Electromagnetic Fields and Neurodegenerative Diseases. Przeglad Elektrotechniczny, 95(1), 129-133. DOI: $10.15199 / 48.2019 .01 .33$

32. Yao A., Zastrow E., Neufeld E., Kuster N. 2019. Efficient and Reliable Assessment of the Maximum Local Tissue Temperature Increase at the Electrodes of Medical Implants under MRI Exposure. Bioelectromagnetics, 40(6), 422-433.

33. Ye D., Xu Y., Wang G., Feng X., Fu T., Zhang H., Jiang L.,Bai Y. 2015. Thermal effects of $2450 \mathrm{MHz}$ microwave exposure near a titanium alloy plate implanted in rabbit limbs. Bioelectromagnetics, 36(4), 309-318.

34. Yee K.S. 1966. Numerical solution of initial boundary value problems involving Maxwell's equations in isotropic media. IEEE Transactions on Antennas and Propagation, 14(3), 302-307.

35. Zradzinski P., Karpowicz J., Gryz K., Leszko W. 2018. Evaluation of the safety of users of active implantable medical devices (AIMD) in the working environment in terms of exposure to electromagnetic fields-Practical approach to the requirements of European Directive 2013/35/EU. International Journal of Occupational Medicine and Environmental Health, 31(6), 795-808.

36. 1999/519/EC: Council Recommendation of 12 July 1999 on the limitation of exposure of the general public to electromagnetic fields $(0 \mathrm{~Hz}$ to $300 \mathrm{GHz})$. Official Journal of the European Communities, L 199, 59-70.

37. 2013/35/EU: Directive 2013/35/EU of the European Parliament and of the Council of 26 June 2013 on the minimum health and safety requirements regarding the exposure of workers to the risks arising from physical agents (electromagnetic fields). Official Journal of the European Union, L 179, 1-21.

38. Duke, 2019, https://itis.swiss/virtual-population/ virtual-population/vip3/duke/

39. Sim4Life, 2019, https://zmt.swiss/sim4life/

40. Thoracic Surgery, 2019, https://thoracickey.com/ surgery-for-pectus-and-other-congenital-chestwall-disorders/ 